\title{
Prosodic Variation in Academic Public Presentations
}

\author{
Elena Freydina \\ Moscow State Pedagogical University \\ Correspondence concerning this article should be addressed to Elena Freydina, Moscow State Pedagogical \\ University, Institute of Philology and Foreign Languages, Prospect Vernadskogo, 88, Moscow, Russian \\ Federation, 119571.E-mail: freydina55@mail.ru
}

\begin{abstract}
The article reports on a study of the prosodic characteristics of academic public presentations. Prosodic variation is analysed with regard to the social and cultural context which is significant for the adequate description of discourse prosody. The paper draws on the findings made in the course of the analysis (auditory and acoustic) of presentations delivered by British lecturers. The article contains an overview of contextual factors and discourse strategies used in academic presentations. Special focus is given to prosodic variations in spoken discourse determined by the extralinguistic context. The author argues that that the choice of prosodic means depends on a variety of contextual parameters: speaker-audience relationships (reflected in the tenor of discourse), the speaker's rhetorical competence, method of delivery, rhetorical tradition and others. The observations made in the paper may be useful to develop expertise in the delivery of academic public presentations which is an important aspect of EFL teachers' professional training.
\end{abstract}

Keywords: prosodic variation, contextual factors, discourse strategies, style, prosodic markers

The search for an adequate description of the prosody of the academic public presentation involves the analysis of the nature of rhetorical discourse and the extralinguistic context including the speech situation and the broader social, cultural and institutional context. This paper is an attempt to combine the study of contextual factors, rhetorical properties and prosodic characteristics of public speeches and to establish correlations between them.

This approach is based on the theoretical assumption that language cannot be considered in isolation from meaning and should be considered within sociocultural context in which it occurs (Halliday, 1978; Paltridge, 2012). It is highly relevant in the study of spoken discourse. Following Brazil (1997, p. 26), "As analysts we cannot hope to make sense of a speaker's behaviour unless we are willing to take into account very much more than is vested with the comparative objectivity of a transcribed text". It should be noted that we must consider both the immediate speech situation ("the here and now" of the utterance, "the local context") and the "broad context" (the social and cultural background).

The studies of spoken academic discourse generally focus on some particular linguistic features: the discourse structure and lexico-grammatical characteristics (Biber, Connor and Upton, 2007), pragmatic and sociolinguistic aspects (Hyland, 2009), rhetorical features (Swales, 2004). The study which drew on the data from undergraduate lectures (Nesi, 2001) looked at the relationship between lexical density and speed of the speech. But on the whole, the prosodic aspect of academic public presentation is seldom included in the analysis despite the fact that it is an essential component of spoken discourse, rhetorical discourse in particular.

The key issue of this research is to describe the prosodic characteristics of the academic presentation with regard to its rhetorical status and sociocultural context and establish the sources of prosodic variation.

\section{Materials and Methods}

The observations presented in this article were made in the course of the research based on the analysis of the academic public presentations delivered by British lecturers (15 men and 10 women) and audio-recorded at the moment of their presentation (25 lectures). 
The topics of the lectures were related to the spheres of education, linguistics, cross-cultural communication, foreign language teaching, social and cultural problems.

Both auditory (perception) analysis and acoustic analysis were used to establish prosodic characteristics of the spoken academic discourse. Auditory analysis was aimed at recording the "auditory impression" of the speech segments and providing their auditory transcription. The objective of the acoustic analysis (Speech Analyzer (v.3.0.1) was to provide measurements of the prosodic parameters: pitch, duration and intensity. Auditory transcription and the data obtained in the course of acoustic analysis of the selected samples made it possible to single out the general tendencies in prosodic variation.

The approach used in this research consisted in combining the analysis of prosodic features and the analysis of contextual factors. This approach leads to an insightful analysis of prosodic variation in spoken discourse and to the understanding of discourse complexity.

\section{Results and Discussion}

There were three steps in the analysis: 1) context analysis (study and description of contextual factors); 2) description of typical prosodic features; 3) interpretation of the relationship between contextual factors and prosodic features in functional terms.

\section{Contextual Factors}

Phonetic research related to "speaking styles", or "phonetic styles" generally focuses on the analysis of prosodic variation brought about by such factors as the goal of the discourse, the degree of formality, and the form of communication (monologue/dialogue). Wichmann bases the analysis of speaking styles on the following parameters: monologue vs. dialogue, public vs. private, goal-oriented vs. unconstrained, scripted vs. unscripted, rehearsed vs. spontaneous (Wichmann, 2000, p. 22). The key problem here is that in each particular case all these and other factors do not act in isolation, but in complex interaction. As a result, the prosodic realisation of the discourse may vary considerably within the same genre.

Looking at the extralinguistic context of the academic public speech (lecture), we can single out a number of factors, the influence of which on prosody has a rather contradictory character. In academic public presentations the choice of prosodic means depends on a variety of contextual parameters. The most obvious among them are these: the speech event is a public monologue, it is goal-oriented.
The goal is to inform, instruct, educate, which involves certain communicative pressure. However, rhetorical discourse is tailored as a two-sided interaction aimed at cooperation between the speaker and the audience. In other words, it is audience centered, which often results in friendly, informal interactions. In fact, in the dynamics of discourse the character of the speaker hearers (audience) relationship reflected in the tenor is of crucial importance in terms of its influence on prosody variation.

The method of delivery chosen by the speaker may also affect the prosodic realisation: manuscript reading, extemporaneous speaking, impromptu method of delivery, memorized method. Basically, prosodic manifestations of the factors related to "mode" (prepared/spontaneous, scripted/ unscripted, monologue/dialogue) are determined by the specific features of rhetorical discourse and are often heterogeneous. Thus, the effect of spontaneity may be deliberately planned by the speaker to maintain contact and make the interaction effective. A lecture is an oral text, but it is generally based on a written text (script), intended for vocal delivery. Such texts characterised as "written to be spoken" are widely used in public presentations. Admittedly, the lecture is a monologue, but elements of dialogue are sometimes incorporated because interactions with the audience and feedback are very common in rhetorical discourse.

Another important factor to consider is described in rhetoric in terms of rhetorical ethos. In other words, it is the broad social and cultural context, rhetorical tradition. British rhetorical tradition is characterised by audience oriented discourse; the speaker avoids being dogmatic; he uses irony, self-irony, respects the personal space of the listeners. In the Russian tradition, lecturers generally tend to be more dogmatic and imposing. As regards spoken academic discourse, the institutional culture and the tradition of the particular university are to be taken into account.

Social variability of intonation is also to be considered. Special focus in this research is given to such factors reflected in the prosodic characteristics of rhetorical discourse as the professional status of the speaker, speaker-audience relationships and the individual style of the speaker. It is generally acknowledged that profession as one of the components of social status affects speech behaviour, and intonation in particular. The lecturer belongs to the group of the so-called "highly verbal" professions which is reflected in the vocal qualities and intonation repertoire. It is also relevant that the teacher or university lecturer represents the authority role which implies a certain amount of discourse control.

It follows from this brief overview of the contextual factors that determine the variation of discourse prosody in academic public presentations that 
the analysis should include the local (situational) context, the broad social and cultural context and the dynamic factors which emerge in the process of the social interaction of discourse participants. These factors regulate the choice of language means, prosodic means in particular, that the speaker makes. The speaker chooses from the speech repertoire what is appropriate. Coupland (2007) compares a speech repertoire to a closet containing a specified number of clothing items. In this conception, speakers select items from their individual clothing (or speech) repertoires. They do that either to match particular situations they find themselves in (situational conformity - dressing 'to fit in'), or to deviate to some extent from normative expectations (initiative style dressing 'to be different') (Coupland, 2007, p. 83).

Considering that public speaking involves both stereotype and creativity, it is necessary to look at the individual style of the speaker as another source of prosodic variation.

\section{Speaker's Strategies and Their Prosodic Realisation}

The contextual factors outlined above in their combination are reflected in the variations of the prosodic characteristics which can be grouped into those related to the goals of educational discourse (instructing, informing) and those related to the sphere of speaker-audience interaction. Correspondingly, the strategies used by the speaker include those aimed at conveying information and interaction strategies aimed at maintaining contact with the listeners (Bloch \& Freydina, 2011, p. 183).

The first group of strategies intended to implement the educational goals of discourse imply active involvement of both the speaker and the audience in the process of communication. A high information potential of the utterance is achieved by a number of prosodic devices (intonation techniques): accentuation in pre-nuclear patterns, increased length of pauses (especially when unexpected information is conveyed), prosodic contrasts and others. The examples below illustrate the use of these intonation techniques:

1. It is $a \searrow$ genuinely 'popular \genre\| (lecture "Thriller as a Literary Genre").

This is the main thesis of the lecture. The material in the thesis which has a high information potential is in broad focus. The whole utterance is perceived as carrying new information.

It should be noted that within the act of argumentation the dominant position of the thesis may be created by means of prosodic contrasts. The phrases preceding and following the thesis are often realized as Low Key information (low pitch level, narrow pitch range, fast tempo, reduced loudness), while the thesis is pronounced as High Key information (high pitch level, broad pitch range, slow tempo, increased loudness). As a result, the material in the thesis acquires greater prosodic prominence.

2. In the written 'language of the \world\| the $\rightarrow$ English of \print $\backslash \backslash$ standard 'English $e \backslash$ xists $\|$ standard 'written $\backslash$ English||| (lecture "The Future of English").

The character of accentuation (word-by-word accentuation) demonstrates that the speaker conveys important information. This device is also associated with "rhetorical pressure".

3. Nearly half occur once||Always||| (lecture "Attraction of Words").

Prosel|lis supposed to be the simplest thing $\|$ (lecture "Stylistics").

Increased duration of pauses between phrases or intonation groups is frequently used when the information is not only new and important, but also unexpected. Prolonged stop of phonation creates anticipation.

4. Personally| I've always taught literature the $\uparrow$ old way\| (lecture "Thriller as a Literary Genre").

The use of Accidental Rise (marked increase of pitch level) emphasises the semantic value of a particular lexical unit. These intonation techniques are often combined. Prosodic prominence of the key elements contributes to forming the information structure of the text and ultimately to getting the information across to the listeners.

The crucial factor in any public speech is the ability of the speaker to build rapport with the audience, to establish and maintain contact and to monitor the reaction of the listeners. Numerous interaction strategies are aimed at expressing the speaker's attitude, facilitating the perception of the speech and maintaining contact.

Two intonation techniques used to implement these strategies are illustrated below.

The first is a high proportion of Fall-Rises on discourse markers in the initial position, adverbs in particular. According to Brazil (1997), "the FallRises used as a referring tone serves to insinuate a measure of generalised intimacy and solidarity into the speaker/hearer relationship - a kind of verbal hand-on your shoulder gesture" (p. 79). In lectures it is a signal of the informal and friendly tenor of discourse.

vAdmittedly| it's hardly a new idea|(lecture "New Developments in Higher Education")

And $\vee$ personally| I am very delighted with it| (lecture "Cambridge") 
Another tendency is that the intonation groups which actualise interaction strategies are contrasted prosodically and perceived as rhetorical signals. Low Key is often used:

And the question is now what happens to a language| when it comes to be used in that kind of wayl and the first thing that happens $\mid$ and this is not always a palatable point| be prepared to be upset||| The first thing that happens| (lecture "The Future of English")

Discourse strategies and prosodic devices (intonation techniques) that actualise them are presented in Table 1.

Table 1

Discourse strategies and intonation techniques in academic public presentations

\begin{tabular}{ll}
\hline Discourse strategies & Intonation devices \\
\hline $\begin{array}{l}\text { Informational } \\
\text { Strategies } \\
\text { information }\end{array}$ & $\begin{array}{l}\text { Word-by-word accentuation } \\
\text { Broad focus position } \\
\text { Contrastive prosody of the } \\
\text { utterance }\end{array}$ \\
$\begin{array}{l}\text { Conveying } \\
\text { important } \\
\text { unexpected facts }\end{array}$ & $\begin{array}{l}\text { Narrow focus } \\
\text { Accidental Rise }\end{array}$ \\
$\begin{array}{l}\text { Change of tempo, } \\
\text { lengthening of pauses } \\
\text { inside the utterance } \\
\text { Contrastive use of nuclear } \\
\text { tone }\end{array}$ \\
\hline
\end{tabular}

\section{Interaction \\ Strategies}

Expressing

the speaker's

attitude

Prosodic prominence

of textual units with

emotional and modal

connotations

\section{Structuring}

the information

and optimizing

its perception by

the listeners
Prosodic prominence of

discourse markers

Fall-Rise on the adverbs in

initial position

Emphatic pauses

Contrast of prosodic parameters: tempo,

Maintaining contact loudness and pitch level

Low Key on contact devices and discourse markers

\section{Prosodic Markers of Style in Academic Public Speech}

The next step of the research consisted in giving a description of typical (pervasive) prosodic features. The data obtained in the course of perception and acoustic analysis demonstrated marked prosodic variations. The explanation of this phenomenon is based upon the analysis of contextual factors and rhetorical characteristics of the academic presentations outlined in 3.1. There can be traced prosodic features associated with the sphere of educational discourse and those that are associated with conversational tenor of discourse. It results from the fact that lectures tend to be organized as an "enlarged conversation" of the speaker and the listeners and are often rather informal.

It will be wrong to assume that the public speech can be identified with everyday conversation. In rhetorical discourse, the speaker controls speech behaviour and resorts to conversational style deliberately using it as a means of building rapport with the audience.

The overall impression is that of the "fusion" of speaking styles which is reflected in heterogeneity of prosodic characteristics (Freydina, 2012, p. 309). The prosodic markers typical of educational rhetorical discourse include the following features: marked variation of pitch ranges (form narrow to wide), frequent use of such terminal tones as High Fall, Fall-Rise, RiseFall-Rise, slow tempo, varied length of pauses, frequent use of emphatic pauses. The markers of informal conversational discourse include: narrow ranges, frequent use of Low Fall, Low Rise, Mid-Level Tone, faster tempo, shorter pauses, presence of hesitation pauses.

It should be noted that conversational tenor is typical of introductions, illustrative examples, commentaries, stories and jokes which are often used in rhetorical discourse. The amount of such fragments depends on the degree of formality, the subject matter and the individual style of the speaker. Since humour and irony are typical of the British tradition of public speaking (Collins, 2012; Fox, 2004), such conversational fragments are often observed:

I'm sorry to be lecturing at the time of dayl when all decent people| should be fast asleep on a halfday\|. I can tell youl it's my habit to sleep at this time\|l. But I am standing $u p \mid$ and it's difficult to sleep standing $u p \|$. But if you nod off $\mid$ I shall understand very well $\|$ (lecture“Thriller as a Literary Genre")

One of the prosodic markers that is generally associated with conversational style is hesitation pause. Admittedly, one would not expect this type of pause to be used in a public speech. It is argued that 
too many hesitation pauses can spoil the effect of the speech as they may be perceived as a marker of the speaker's uncertainty and lack of confidence. Besides, pausing in the wrong place can change the meaning or make the message unclear. Contrary to these recommendations of specialists in public speaking, the research demonstrated a considerable number of hesitation pauses (both silent and filled) in academic public presentations.

The use of hesitation pauses in lectures is determined by the fact that they can perform a variety of functions: they may be used for speech planning (self-repair, finding appropriate wording), they may reflect the physiological and psychological state of the speaker, they may serve as a contact device. In academic public presentations, hesitation pauses may serve as signals of informal, friendly and spontaneous tenor of discourse.

It should be mentioned here that to identify the function of the hesitation pause one must rely on the context of the speech as well as the competence and individual style of the speaker:

I mean different meanings of words $\mid$ different words $\mid$ er | spellings of words $\mid$ (self-correction). (lecture "Attraction of Words")

But prose $\mid$ is not it's the most difficult to er | reproduce $\|$ You haven't got poetic license $\|$ You've got to|er|do justice| to the author's intent|| (lack of rhetorical competence).(lecture "Stylistics")

And $\mid$ er|in fact| common observation| if you look around people who read | good fiction | shows you| that they are not better people| than people who read bad fiction $\|$ (signal of informal interaction). (lecture"Thriller as a Literary Genre")

Another variable that serves a marker of style is tempo. Slow tempo is used to convey important information; it also indicates a high degree of rhetorical pressure. Faster tempo is used in conversational fragments (illustrative examples and anecdotes). On the whole, a marked variation of tempo contributes to making the discourse dynamic and, ultimately, contributes to its effectiveness.

Pitch parameters also serve as markers of "the fusion of styles". The most important are the character of the terminal tone and variations of pitch range. Cruttenden (1997) made the following observation about the stylistic aspect of nuclear tones:

of the four tones which are most typical of sentence non-final intonation groups (Low Rise, High Rise, Fall-Rise and Mid-Level), two (Low Rise and Fall-Rise) are more typical of formal styles.
Of the two typical of informal styles, High Rise is the particularly 'casual one' whereas Mid-Level, while being a common non-final tone in conversation, is also common in the speeches of politicians who pride themselves on possessing the common touch (Cruttenden, 1997, p. 128).

In the lecture, the terminal tones associated with formal discourse are traced alongside with those that are associated with informal interaction which makes it difficult to identify and describe the intonation patterns typical of this genre of discourse.

In some fragments, pitch characteristics are similar to those in spontaneous discourse: narrow pitch range, prevalence of Mid-Level Tones and Low Falls, Mid and Low Level Tones in pre-nuclear parts of the intonation groups.

I used to have a box by the door and instead of throwing away all my junk mail without opening it|I used to put it into the box so it could go into the corpus||. Language of the e-mails $\|$. We've got a small e-mail corpus $\mid \|$. (lecture "Attraction of words")

Outside the context of the public speech, such fragments could be perceived as parts of spontaneous monologue; however, they are integral components of the present-day academic presentation.

Some of the markers of style heterogeneity and prosodic variability are presented in Table 2 .

It is clearly evident that the traditional prosodic markers of academic style predominate. But my primary concern here was to show that the description of the prosodic features of the lecture cannot be restricted to a fixed set of prosodic parameters. Such an inventory of prosodic features is not sufficient and does not reflect the discourse complexity. The nature of rhetorical discourse and its dynamic character determine extensive prosodic variability.

\section{Prosodic Markers of Individual Style}

Another source of prosodic variation in the academic public presentation is the individual style of the speaker. It is generally acknowledged that one of the most important aspects of good public speaking is the personality the speaker projects. On the one hand, public speech is guided and restricted by the rhetorical tradition with rules prescribed for each step of public speaking. On the other hand, each public presentation is a unique "rhetorical event", which reflects the personality of the speaker and the persona he projects.

The markers of the speaker's identity in academic discourse are a reflection of a complex set of factors: physiological and psychological, social (age, gender, 
Table 2

Prosodic markers of style

\begin{tabular}{|c|c|c|}
\hline Prosodic features & Academic style & Conversational style \\
\hline Key & $\begin{array}{l}\text { Greatly varied, mostly high and } \\
\text { medium }\end{array}$ & Low, medium \\
\hline Pitch ranges & $\begin{array}{l}\text { Greatly varied, mostly broad and } \\
\text { medium }\end{array}$ & Medium, narrow \\
\hline Terminal tones & $\begin{array}{l}\text { High Falls, Mid Falls, Fall-Rises, } \\
\text { compound tones }\end{array}$ & $\begin{array}{l}\text { Mid Level Tones, Low Falls, Fall- } \\
\text { Rises }\end{array}$ \\
\hline $\begin{array}{l}\text { Pre-nuclear patterns } \\
\text { (Heads) }\end{array}$ & $\begin{array}{l}\text { High Level Head, Falling Head, } \\
\text { Stepping Head, Sliding Head }\end{array}$ & $\begin{array}{l}\text { Low Level Head, Mid Level Head, } \\
\text { Falling Head }\end{array}$ \\
\hline Segmentation & $\begin{array}{l}\text { Medium and long intonation } \\
\text { groups }\end{array}$ & $\begin{array}{l}\text { Varied length of intonation } \\
\text { groups with a high proportion } \\
\text { of short intonation groups }\end{array}$ \\
\hline Rate of speech & $\begin{array}{l}\text { Average, decreasing on important } \\
\text { parts of utterance }\end{array}$ & Fast \\
\hline Pauses & $\begin{array}{l}\text { Syntactic pauses, medium and } \\
\text { long: emphatic and rhetorical } \\
\text { pauses }\end{array}$ & $\begin{array}{l}\text { Considerable number } \\
\text { of hesitation pauses (filled and } \\
\text { unfilled) }\end{array}$ \\
\hline
\end{tabular}

social and educational background), the level of professional competence (academic, rhetorical), attitude to the subject of the presentation (interested/ uninterested, involved/ uninvolved) and attitude to the audience. Under the influence of these factors, the individual speech repertoire is formed.

Admittedly, phonetic means contributes to creating the personal style of the speaker. The comparative analysis of text samples from the corpus of research showed that the following prosodic parameters can serve as indicators of individual style: the degree of variability of pitch ranges, preference of particular intonation patterns in final and non-final positions, variability of tempo, rhythmic organisation, frequency of emphatic, rhetorical and hesitation pauses, and variability of loudness. Vocal qualities are also relevant: voice timbre, voice qualities, tone of voice and voice modulations

On the whole, the individual style is a unique combination of segmental, suprasegmental, paralinguistic features.

\section{Conclusion}

The results of the research presented in this article demonstrate the dimension of prosodic variations in academic public presentations. Studying discourse prosody with regard to the sociocultural context enables the analyst to get some interesting insights concerning both the use of language, prosodic means in particular, and social and cultural parameters of speech interaction: participants of discourse, their relationship, social and cultural values, and rhetorical tradition.

The complexity of academic spoken discourse is reflected in extensive prosodic variation. In both academic spoken and written interactions, academic and conversational styles may intermingle with each other. The prosodic markers of this heterogeneity are the character of text segmentation, marked variation of tempo, the character of pauses and pitch parameters. It should be noted that marked prosodic variation contributes to making the public presentation more dynamic and expressive. Thus, it contributes to the effectiveness of rhetorical discourse.

Admittedly, expertise in the delivery of academic public presentations is an important aspect of the professional training of EFL teachers. The observations made here are relevant in terms of teaching the skills of public speaking to Russian learners of English and developing the understanding of the specific features of the present day academic presentation. 


\section{References}

Biber, D., Connor, U., \& Upton, T. (2007). Discourse on the move: Using corpus analysis to describe discourse structure. Amsterdam, Netherlands: John Benjamins.

Bloch, M., \& Freydina, E. (2011). Public speech and its prosodic organisation. Moscow, Russia: Prometey.

Brazil, D. (1997). The communicative value of intonation. Cambridge, UK: Cambridge University Press.

Collins, P. (2012). The art of speeches and presentations: The secret of making people remember what you say. The Atrium Southern Gate, Chichester, UK: Wiley and Sons Wiley.

Coupland, N. (2007). Style: Language variation and identity. Cambridge, UK: Cambridge University Press.

Cruttenden, A. (1986). Intonation. Cambridge, UK: Cambridge University Press.

Fox, K. (2005). Watching the English. The hidden rules of English behaviour. London, UK: Hodder \& Stoughton.
Freydina, E. (2012). Prosodija kak faktor stilevogo var'irovanija zvuchashhej rechi [Prosody as a factor of style variation in spoken discourse]. Prepodavatel XXI Century, 3(2), 307-313.

Halliday, M. A. K. (1978). Language as social semiotic: The social interpretation of language and meaning. London, UK: Edward Arnold.

Hyland, K. (2009). Academic discourse. London, UK: Continuum.

Nesi, H. (2001). A corpus-based analysis of academic lectures across disciplines. In J. Cotteril, A. Ife (Eds.), Language across boundaries (pp. 201-218). London, UK: BAAL in association with Continuum Press.

Paltridge, B. (2012). Discourse analysis. London, UK: Bloomsbury Academic.

Swales, J. M. (2004). Research genres: Explorations and applications. Cambridge, UK: Cambridge University Press.

Wichmann, A. (2000). Intonation in text and discourse. Beginnings, middles and ends. Harlow, UK: Pearson Education Limited. 